\title{
Lumped, constrained cable modeling with explicit state-space formulation using an elastic version of Baumgarte stabilization
}

\author{
Stian Skjong \\ Research Scientist \\ Department of Seafood Technology \\ SINTEF Ocean \\ Email: stian.skjong@sintef.no
}

\author{
Karl Johan Reite \\ Research Scientist \\ Department of Seafood Technology \\ SINTEF Ocean \\ Email: karl.j.reite@sintef.no
}

\author{
Karl Gunnar Aarsæther \\ Associate Professor \\ Department of Technology and Safety \\ UiT The Arctic University of Norway \\ Email: karl.g.aarsather@uit.no
}

This paper presents a modeling approach for efficient simulation of slender structures, such as wires, cables and ropes. Lumped structural elements are connected using constraints. These are solved explicitly, using an elastic version of Baumgarte stabilization. This avoids singularities in the matrix inversions. The resulting explicit state-space formulation filters the higher order dynamics and can be solved using simple numerical integration methods. Constraints are demonstrated for modeling different aspects: Internal cable forces, one cable sliding along another cable and contact between cable and seabed. Also, a cable initialization routine is presented for rapid building of different interconnected cable geometries, ranging from cases in offshore crane operations to in-sea equipment such as seismic cables. Two case studies are presented to illustrate the effectiveness and the robustness of the proposed modeling approach; the first one being a test of two connected, sinking cables, and the last one being a larger case demonstrating the use of the cable library in an offshore seismic survey case.

\section{Introduction}

Wires, cables and ropes are widely used in industry. In the offshore maritime segment, they are used in areas of application ranging from anchor lines, fishing gear, lifting- and towing operations and seismic surveys. Mathematical models for cables and wires are often present in operational planning analyses based on dynamical simulations with models representing the various system components. Depending on the type of analysis, models with suited fidelity levels are used to represent the required characteristics of the system. For example, if the goal of the analysis is to study the fatigue strength of a cable, as done in [1] for flexible umbili- cal members for marine structures, a model based on a $\mathrm{Fi}$ nite Element Method (FEM) is typically used. Such models are computationally intensive, leading to simplified modeling or long computation times. More computational efficient methods are therefore wanted for time-restricted modeling of the global forces and movements of slender structures. This is the scope of this article. On-line decision support systems may be used to give the operator a window into the hidden, but mathematically observable, system behavior and require efficient and robust numeric methods for implementation. Calculation of system dynamics driven by measurements surpass the human ability to predict motions of complex interconnected systems, which are frequently encountered in operations of marine equipment. Effects of changes to the system configuration, and "what if" questions, can be presented to, and evaluated by, the operator with a re-configurable model with predictive power. This paper presents a computationally efficient, and numerically robust method, suitable for implementation of on-line decision support systems for the interconnected cable systems found in marine equipment.

Real-time capable simulation models used for system surveillance and state estimation purposes are often referred to as digital twins. There exists a few modeling methods for flexible, long and slender structures with real-time applicable potentials. One such method is modal system modeling using a sum of normal modes to describe the geometry of the structure based on given boundary conditions, material characteristics, loading conditions and energy considerations [2]. Modal theory has been used to model various systems, ranging from beam structures and rotor dynamics [3, 4] to hydraulic transmission lines [5] and acoustic filters [6]. This method results in a compact state-space formulation of the 
system, where the number of states are related to the number of normal modes included in the model. A limited number of modes are typically needed to achieve results of the desired accuracy [4]. The derivation of the modal state-space model is, however, strongly affected by the loading conditions, the type of forces acting on the body and the boundary conditions. This implies lack of flexibility for modeling systems that vary in structure during the period of interest. A particular case is the response from forces attacking in a dynamic point, which is the case for sliding constraints that will be discussed later. Flexibility that allows for definition of interconnected cables is needed for a general cable formulation which may be applied in real-time simulations of the previously mentioned marine operation. The modeling approach and constraints formulation presented in Sec. 2.4 is sufficient in order to implement real-time models for a wide variety of offshore maritime operations.

A mass-spring-damper model is often used for representing slender structures. Using this approach, the structural mass is lumped between discrete mass nodes distributed throughout the the structure and interconnected by a network of springs and dampers. These models are simple to implement and to understand, but cannot represent bending and torsion without interconnected springs in different element directions, such as shown in [7], which increase complexity of implementation and modeling. Stiff springs may be required to reflect the material properties. This may introduce high eigenfrequencies in the system and numerical oscillations caused by the solver dynamics. This leads to reduced simulation time-step sizes, increased computational effort and longer solution time which are undesirable in online decision support applications. This can to some extent be alleviated by coarse models, at the expense of fidelity. Many examples of lumped cable and net models are found in the literature, such as [8, 9, 10]. In [11] a FEM model of offshore seismic cables are compared with a lumped parameter model, consisting of linear mass-spring-damper arrangements, with good results. In [12] a lumped model of an offshore crane-wire was modeled in 3D based on the 2D model of a winch wire presented in [13].

Instead of using a spring-damper arrangement between each lumped element, one can define a set of constraints that serves the same purpose. However, this will lead to a system of differential-algebraic equations, but by defining the constraint convergence dynamics the result is a set of differential equations of system and constraint dynamics. The constraint convergence dynamics may be related to material- and system characteristics and the constraints are included explicitly in the state-space formulation of the system as forces [14], this will be discussed in detail in Sec. 2.1. Hence, numerical oscillations can be repressed by the choice of suitable constraint dynamics, or constraint control laws. This method for handling constrained dynamics is referred to as Baumgarte stabilization, which in general uses analytical reformulations of constraint forces in mechanical systems to handle differential algebraic equations [15]. Reformulated models of differential equations can be solved using standard integration routines, including the explicit Euler method used in this ar- ticle. This method performs well on stiff systems, as shown in [16, 17].

The use of constraints to lump elements of a structure together does not put any restrictions on the boundary conditions, the model environment, the model connectivity or the type of forces acting on each element. Hence, this modeling approach has the potential of generating modular, generic models which can be used in on-line simulation connected to various real-time systems. A particular advantage is the ability to decide the speed of the constraint dynamics, effectively filtering out from the solver high frequency motions and gives predictable step size and computational effort. It is, however, important that the constraint dynamics are significant faster than that of important system behavior. Another issue with this method is that acceleration terms introduced by the constraint dynamics can lead to numerical singularities related to matrix inversions. This will be discussed in more detail later. This work uses an elastic version of the Baumgarte stabilization method to overcome this problem. The method presented herein, results in a mass matrix that is always invertible and numerically well-conditioned. This gives the method the possibility of applying iterative methods for matrix inversion which increase the efficacy of the method.

In this work, co-simulation and the Functional Mockup Interface standard (FMI) will be used as the simulation method and the model interface standard, respectively, ensuring a standardized and modular formulation of the model interfaces. Co-simulation technology will not be discussed further, but the reader should be aware of its implications. The approach of co-simulation is to split a systems simulation into several subsimulators, solve them in parallel and only exchange information at certain times. Hence, there is an assumption that each subsimulator is loosely coupled with the rest of the simulation. Also, the stability of the simulation results might depend on the different time steps in the co-simulation. For more details about co-simulations, the reader is referred to [18, 19]. Nevertheless, co-simulations has been capable of interacting with real-time systems, e.g. providing measurements from a physical system to a realtime algorithm such as a control system, and to feed back the control action to the physical system [20].

This article presents a lumped element cable library for modeling cables, ropes and wires in maritime applications with real-time capabilities. This library use the elastic Baumgarte stabilization method to avoid singularities and to enable explicit formulations of constraint forces which can be solved by any numerical integrator. Moreover, this article unifies the preliminary results presented in [21, 22] and provides a novel discussion of common constraints needed to model slender, flexible marine structures. Hence, the work presented in this article contributes towards robust, modular and real-time capable simulation models of various cable configurations, for the purpose of analyzing marine operations and for designing digital twins for various flexible and slender equipment used in different marine operations. 


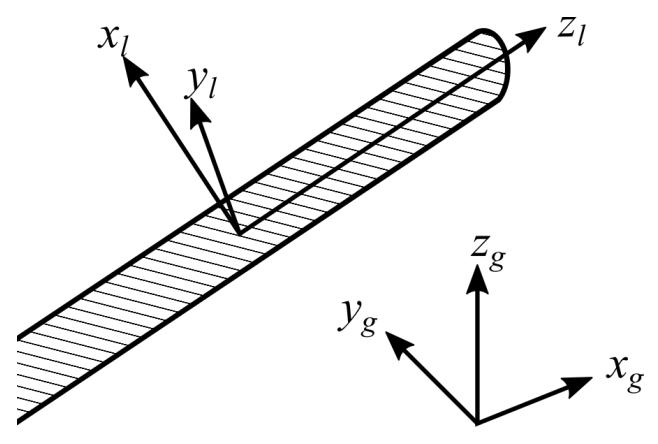

Fig. 1: Cable element with local v.s. global coordinate frames

\section{Modeling}

In this work the following vector and matrix syntax will be used for the lumped models. A vector $\mathbf{p}$, being e.g. a position vector, velocity vector or acceleration vector, for lumped element $i$ in model $j$ in reference frame $a$ at the lumped body point $b$, is given as ${ }_{i}^{j} \mathbf{p}_{a / b}$. Following, a rotation matrix $\mathbf{R}$ for lumped element $i$ in model $j$ rotating from reference frame $b$ to $a$ is expressed as ${ }_{i}^{j} \mathbf{R}_{a / b}$. Other definitions are also used for readability reasons, and will be explained where first used. Also, bold letters denote vectors or matrices.

The global reference frame, here referred to as the $N W U$-reference frame, is defined such that the $x$-axis points to the north, the $y$-axis towards west and the $z$-axis upwards, being zero at the water surface. The local reference frame for a lumped cable element is defined such that the $z$-axis is pointing along the element itself. Both the global and the local reference frames are illustrated in Fig. 1]

\subsection{The basic model}

The basic cable model is composed of a given number of connected elements, where each element is modeled as a bar, as shown in Fig. 1 In general, the dynamics for an element $i$ can be expressed as

$$
\begin{aligned}
\dot{\mathbf{x}}_{i} & =\mathbf{v}_{i}, \\
\mathbf{M}_{i} \dot{\mathbf{v}}_{i} & =\mathbf{F}_{i}+\mathbf{F}_{c, i},
\end{aligned}
$$

where $\mathbf{x}_{i}$ is the position and Euler angles, $\mathbf{v}_{i}$ is a state vector holding the velocities and Euler angle rates, $\mathbf{M}_{i}$ is the mass matrix, and $\mathbf{F}_{i}$ is a vector holding the forces and torques acting on the element (such as gravitational forces). $\mathbf{F}_{c, i}$ is the constraint force vector which contains forces used to constrain the system, e.g. for linking the elements together according to the model description. These constraints are predefined and can be solved explicitly from the constraint force vector

$$
\mathbf{F}_{c}=-\mathbf{J}^{\top} \lambda,
$$

where $\mathbf{J} \in \mathbb{R}^{\text {size }(\mathbf{C}) \times \operatorname{size}(\dot{\mathbf{x}})}$ is the partial derivative of the constraint vector $\mathbf{C}(\mathbf{x}, \mathbf{v})=\mathbf{0}, \mathbf{C} \in \mathbb{R}^{n c}$ where $n c$ is the number
Table 1: Relations between $\beta$ and material properties. Note that $E$ is the modulus of elasticity, $A$ is the cross section area, $I$ is the area moment of inertia, $G$ is the rigidity material constant, and $J$ is the torsion constant.

\begin{tabular}{|l|l|}
\hline Expression & Material property \\
\hline$\beta^{2}=E A \varepsilon$ & Axial stiffness \\
$\beta^{2}=E I \varepsilon$ & Bending stiffness \\
$\beta^{2}=G J \varepsilon$ & Torsional stiffness \\
\hline
\end{tabular}

of constraints in the system, and $\lambda$ is the Lagrange multiplier. To calculate $\lambda$ the Baumgarte stabilization method [14] can be used where the stabilization law is defined as

$$
\ddot{\mathbf{C}}+2 \alpha \dot{\mathbf{C}}+\beta^{2} \mathbf{C}=\mathbf{0}
$$

where $\alpha$ and $\beta$ are tuning variables, either scalars or diagonal matrices. Note that $\beta$ can be related to physical characteristics, e.g. as material properties, as shown in Table 1 . For a common $\beta$ value, the relationships in the table shows how the epsilon value can be selected for each degree of freedom to preserve the elastic properties of the element.

By using

$$
\dot{\mathbf{C}}=\frac{d \mathbf{C}}{d \mathbf{x}} \frac{d \mathbf{x}}{d t}=\mathbf{J} \dot{\mathbf{x}} \quad \text { and } \quad \ddot{\mathbf{C}}=\dot{\mathbf{J}} \dot{\mathbf{x}}+\mathbf{J} \ddot{\mathbf{x}}
$$

and combining Eq. (1) and Eq. (3), we obtain the total system of interconnected cable elements expressed as

$$
\begin{aligned}
\dot{\mathbf{x}} & =\mathbf{v} \\
\mathbf{M} & =\mathbf{F}_{e x t} \\
& -\mathbf{J}^{\top}\left(\mathbf{J M}^{-1} \mathbf{J}^{\top}\right)^{-1}\left(\mathbf{J M}^{-1} \mathbf{F}+\mathbf{J} \mathbf{v}+2 \alpha \mathbf{J v}+\beta^{2} \mathbf{C}\right)
\end{aligned}
$$

\subsection{Elastic Baumgarte stabilization}

One of the requirements in Eq. (5) is that the mass matrix is invertible, avoiding numerical errors due to singularities. Since this cannot be guaranteed in a real-time applicable, general simulation model involving cables where iterative matrix inversion procedures are often applied, an elastic version of the Baumgarte stabilization is used [22, 21], assuring a positive definite inertia matrix. This changes the constraint dynamics given in Eq. (3) to

$$
\ddot{\mathbf{C}}+2 \alpha \dot{\mathbf{C}}+\beta^{2} \mathbf{C}=\varepsilon \mathbf{I} \lambda
$$

where $\alpha$ and $\beta$ are tuning variables, either scalars or matrices, and $\varepsilon$ is a small positive number, representing the elastic part of the method. By combining Eq. (1), Eq. (4) and Eq. (6), the total system of connected elements can be expressed as

$$
\begin{aligned}
\dot{\mathbf{x}} & =\mathbf{v}, \\
\left(\mathbf{M}+\mathbf{J}^{\top} \Gamma \mathbf{J}\right) \dot{\mathbf{v}} & =\mathbf{F}-\mathbf{J}^{\top} \Gamma\left(\mathbf{J} \mathbf{v}+2 \alpha \mathbf{J} \mathbf{v}+\beta^{2} \mathbf{C}\right),
\end{aligned}
$$


where $\Gamma=\varepsilon^{-1} \mathbf{I}$.

To also avoid singularities introduced by the Euler angles, quaternions are used for the orientations. This changes the state space formulation to

$$
\begin{aligned}
\dot{\mathbf{x}} & =\mathbf{H}(\mathbf{x}) \mathbf{v}, \\
\left(\mathbf{M}+\mathbf{H}^{\top} \mathbf{J}^{\top} \Gamma \mathbf{J H}\right) \dot{\mathbf{v}} & =\mathbf{F} \\
& -\mathbf{H}^{\top} \mathbf{J}^{\top} \Gamma\left(\dot{\mathbf{J} H \mathbf{v}}+\mathbf{J} \dot{\mathbf{H}} \mathbf{v}+2 \alpha \mathbf{J H} \mathbf{v}+\beta^{2} \mathbf{C}\right),
\end{aligned}
$$

where $\mathbf{H}(\cdot)$ contains the mapping between rotation quaternions and Euler orientation rates. Note that the angle rates (in $v$ ) are still expressed through Euler angle rates.

The equations given in Eq. (8) shows a general state-space formulation of mass-elements lumped together through well-defined constraints, potentially being affected by environmental- and external forces. Such constraints and forces are elaborated in the following.

\subsection{Other element types}

Even though the main focus here is devoted to cable dynamics, some elements need to control the boundary conditions for the cables, such as controlling the characteristics of the cable ends. Three different types will be presented here, namely a "passive" element taking position commands as inputs, an "active" element having physical properties, e.g. a buoy, and a hybrid between the two, e.g. a position controlled buoy with tension saturation characteristics.

\subsubsection{Position-controlled points}

Here, the passive position controlled element is an element with no geometry and is only used to control the end of a cable. It takes a position $\mathbf{p}$ as input, and employs a second order normalized Butterworth low-pass filter to estimate its positions and velocities:

$$
\begin{aligned}
& \dot{\mathbf{x}}=\mathbf{v} \\
& \dot{\mathbf{v}}=-\mathbf{x}-\sqrt{2} \cdot \mathbf{v}+\mathbf{p}
\end{aligned}
$$

where $\mathbf{x} \in \mathbb{R}^{3}$ is the position states and $\mathbf{v} \in \mathbb{R}^{3}$ is the velocity states. Note that there are no orientations for this type of element. The second order filter used to estimate the element dynamics is required by the procedure of solving the constraint vectors, as discussed in Sec. 2.2 Note that no external or constraint forces $(\mathbf{J}=\mathbf{0})$ are given to the state equations.

\subsubsection{Passive points}

The passive element type is a point type that has a geometry and a mass, and movements are only affected by modeled external forces. Its state-space model is as given in Eq. (8). In this work, this point type is used for representing buoys influenced by hydrodynamic forces, gravity and buoyancy, in addition to possible constraint forces. This will be elaborated in Sec. 2.5.

\subsubsection{Hybrid points}

The hybrid point is a point type that has the characteristics of the passive point type, but in addition is controlled based on a time varying position input. The position input is transformed to control forces, which are saturated based on tension measurements from the element connected to it.

\subsection{Constraint types}

The types of constraints added to a cable set the limits and freedoms for the cable geometry. In many cases physical effects can be represented either as constraints or as external forces. This is typically the case for friction forces. To illustrate how to define the constraint vector in different cases, a few constraint types are presented in the following. Note that all constraints are here given in the global reference frame.

\subsubsection{Fixed position constraints}

A fixed-end constraint is a constraint for fixing the end of a lump-segment, e.g. as the one shown in Fig. 1. to an external position (either static or dynamic) or another segment. The constraint law itself should represent the material stiffness properties, as explained in Sec.2.2. Note that a segment here is not only considering a cable element, it can be other mass elements with defined geometrical properties, such as e.g. a buoy as elaborated in Sec. 2.3. In general, fixing a position 1 to a position 2 produce the following constraint

$$
\mathbf{C}_{p o s}={ }_{1} \mathbf{p}_{g}-{ }_{2} \mathbf{p}_{g}=\mathbf{0}
$$

where ${ }_{i} \mathbf{p}_{g} \in \mathbb{R}^{3}$ denotes position $i$ in the global reference system $(g)$. If we were linking the end of a cable segment 1 to a fixed global position 2, then ${ }_{1} \mathbf{p}_{g}$ in Eq. (10) would change to

$$
{ }_{1} \mathbf{p}_{g}={ }_{1} \mathbf{p}_{g / c g} \pm{ }_{1} \mathbf{R}_{g / l}\left[\begin{array}{c}
0 \\
0 \\
\frac{L_{1}}{2}
\end{array}\right]
$$

where ${ }_{1} \mathbf{p}_{g / c g} \in \mathbb{R}^{3}$ is the global position of the center of gravity of segment $1,{ }_{1} \mathbf{R}_{g / l} \in \mathbb{R}^{3 \times 3}$ is the rotation matrix for transforming a local vector in the body fixed reference system of segment 1 to the global reference system and $L_{1}$ is the length of the cable segment 1 . Note that \pm is used in the equation to illustrate the difference when working with one end of the cable segment or the other, as also in Fig. 1. Note that if two succeeding cable segments are linked together (a cable segment 1 to a cable segment 2), ${ }_{2} \mathbf{p}_{g}$ would be expressed similar to ${ }_{1} \mathbf{p}_{g}$ and the resulting constraint would be

$$
\mathbf{C}_{p o s}={ }_{1} \mathbf{p}_{g / c g} \pm{ }_{1} \mathbf{R}_{g / l}\left[\begin{array}{c}
0 \\
0 \\
\frac{L_{1}}{2}
\end{array}\right]-{ }_{2} \mathbf{p}_{g / c g} \mp{ }_{2} \mathbf{R}_{g / l}\left[\begin{array}{c}
0 \\
0 \\
\frac{L_{2}}{2}
\end{array}\right]=\mathbf{0}
$$

For succeeding cable elements used to model the same cable also bending stiffness constraints should be considered, as is explained in the following. 


\subsubsection{Bending- and torsion constraints}

An angle constraint between two segments 1 and 2 can be expressed as

$$
C_{\measuredangle}=\theta_{1}-\theta_{2}=0
$$

where $\theta_{1}$ and $\theta_{2}$ represent an angle in segment 1 and 2, respectively. If bending (and torsion) stiffness are considered in all degrees of freedom, the constraints would be expressed as

$$
\mathbf{C}_{\measuredangle}=\Theta_{1}-\Theta_{2}=\mathbf{0}
$$

where $\Theta_{i} \in \mathbb{R}^{3}$ is the vector of Euler-angles for the lumped segment $i$. However, if we are using quaterions, $\mathbf{q}$, the constraints change to

$$
\mathbf{C}_{\measuredangle}=\mathbf{q}_{1}^{-1} \mathbf{q}_{2}-\mathbf{q}_{0}=\mathbf{0}
$$

where $q_{i}$, is the quaternions for lumped segment $i$ and where $\mathbf{q}_{0}=[1,0,0,0]^{\top}$.

\subsubsection{Depth constraints}

In marine operations, depth constraints can be useful to implement seabed interactions. The first step is to identify the deepest end of each lumped segment and test if it violates the seabed restriction. If the different ends of the lumped cable segment are referred to as $A$ and $B$, the depths of the two ends for a segment $i$ are given as

$$
{ }_{i} z_{g / A},{ }_{i} z_{g / B}={ }_{i} z_{g / c g} \pm[0,0,1]^{\top}{ }_{i} R_{g / l}\left[\begin{array}{c}
0 \\
0 \\
\frac{L_{i}}{2}
\end{array}\right]
$$

By defining $i z_{g / \min }=\min \left(i z_{g / A}, i_{g / B}\right)$, and since the global $z$-axis is pointing upwards* the constraint can be expressed as

$$
C_{\curvearrowleft}=\left\{\begin{array}{cc}
i z_{g / \text { min }}-{ }_{\text {depth }} z_{g}= & 0 \text { if } i z_{g / \min } \leq{ }_{\text {depth }} z_{g} \\
0 & \text { else }
\end{array}\right.
$$

Constraints could also be added to model the friction between the non-elastic cable segment, which are linked together through elasticity constraints, and the seafloor when $i z_{g / \min } \leq{ }_{\text {depth }} z_{g}$, similar to the friction forces derived in the sliding constraint, see Eq. 19)-Eq. 21.

\subsubsection{Sliding-end constraint}

Assume that the start of cable 1, element 1, slides along cable 2, as illustrated in Fig. 22 The element of cable 2 which

\footnotetext{
* since we are using the NWU reference system convention.
}

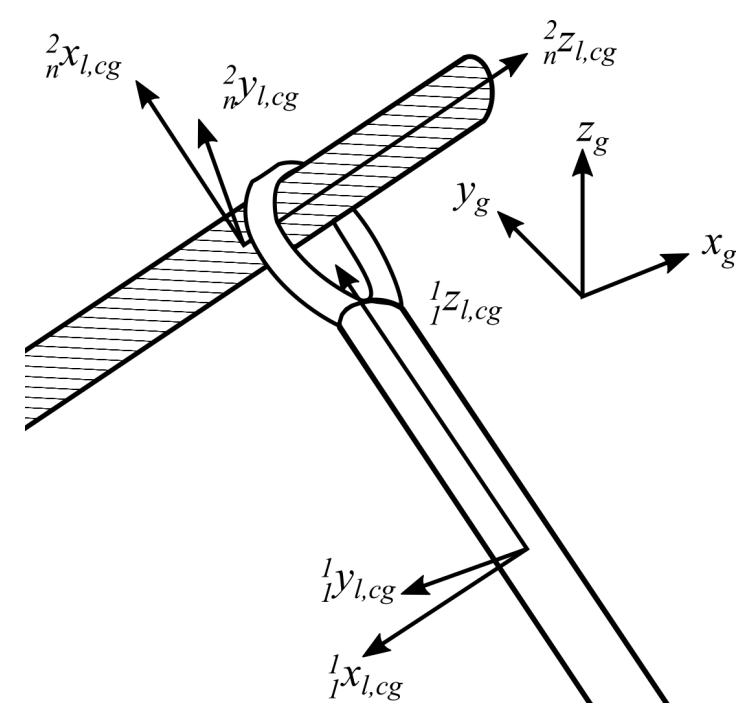

Fig. 2: Sliding cable constraint. The end of segment 1 of cable 1 is sliding alongside segment $n$ of cable 2

is closest to cable 1 must be identified. This is a trivial task and is assume here as element $n$ of cable 2. Also, for simplicity reasons, it is assumed that the end $A$ of segment 1 in cable 1 slides along cable 2 (local z-direction for segment $n$ in cable 2). Hence, the constraints needed for making the end of cable 1 slide along cable 2 are given as

$$
\mathbf{C}_{-\circ}^{\top}=\left({ }_{n}^{2} \mathbf{p}_{g / c g}-{ }_{1}^{1} \mathbf{p}_{g / A}\right)_{n}^{\top 2} \mathbf{R}_{g / l}\left[\begin{array}{ll}
1 & 0 \\
0 & 1 \\
0 & 0
\end{array}\right]=\mathbf{0}
$$

Note that axial stiffness properties can be used for the value of $\beta^{2}$ for sliding constraints. Friction between the two cables can be included by adding a constraint for the difference in velocity between the two cables in the local $\mathrm{x}$-direction for cable 2. This gives the constraint

$$
C \leftrightharpoons=\Delta_{n}^{2} v_{l}=\left[{ }_{n}^{2} \mathbf{R}_{g / l} \frac{d}{d t}\left({ }_{n}^{2} \mathbf{p}_{g / c g}-{ }_{1}^{1} \mathbf{p}_{g / A}\right)\right]^{\top}\left[\begin{array}{l}
0 \\
0 \\
1
\end{array}\right]=0
$$

The sliding friction parameter ${ }^{\dagger}$ is set as $\beta^{2}=\varepsilon^{-1}$ and the value of $\Gamma$ is adjusted related to the sliding friction constraint, here denoted as $\Gamma \leftrightharpoons$,

$$
\Gamma \leftrightharpoons=\mu \frac{\left|F_{N}\right|}{1+\varepsilon^{-1}\left|\Delta_{n}^{2} v_{l}\right|}
$$

where $F_{N}$ is the normal force of the sliding constraint, e.g. given as

$$
F_{N}=\beta^{2} \sqrt{\mathbf{C}_{-}^{\top} \mathbf{C}_{\multimap}}=\beta^{2}\left\|\mathbf{C}_{-}\right\|_{2}
$$

where $\mathbf{C}_{-}$is given as in Eq. 18 .

\footnotetext{
$\dagger$ according to the sliding friction law $F_{f}=\mu\left|F_{N}\right|$ where $F_{N}$ is the normal force orthogonal to the direction of motion.
} 


\subsection{Additional forces}

In addition to the constraint forces other forces may act on the cable system as well. Some of these forces are considered external, such as gravity and hydrodynamic drag, while others are considered internal, such as Coriolis forces. Note that all forces are here presented in the local, body-fixed reference frame.

\subsubsection{Coriolis forces}

For each lumped cable element, the Coriolis and centripetal forces, under the assumption that both the mass matrix and the rotational inertia matrix are diagonal, are given as

$$
\mathbf{F}_{v \omega, i}=\left[\begin{array}{c}
-\left(\mathbf{M}_{i} \cdot{ }_{i} \mathbf{v}_{l / c g}\right) \times \omega_{i} \\
-\left(\mathbf{M}_{i} \cdot{ }_{i} \mathbf{v}_{l / c g}\right) \times{ }_{i} \mathbf{v}_{l / c g}-\left(\mathbf{J}_{i} \omega_{i}\right) \times \omega_{i}
\end{array}\right]
$$

where $\mathbf{F}_{v \omega, i} \in \mathbb{R}^{6}$ is the force and torque vector, $\mathbf{M}_{i} \in \mathbb{R}^{3}$ is the mass matrix for element $i, \mathbf{J}_{i} \in \mathbb{R}^{3}$ is the rotational inertia matrix for element $i,{ }_{i} \mathbf{v}_{l / c g}$ is the velocity vector for element $i$ and $\omega_{i}$ is the Euler angle rates vector for element $i$. These forces can according to [23] also be expressed as

$$
\mathbf{F}_{v \omega, i}=\left[\begin{array}{cc}
\mathbf{0} & -S\left(\mathbf{M}_{i} \cdot{ }_{i} \mathbf{v}_{l / c g}\right) \\
-S\left(\mathbf{M}_{i} \cdot{ }_{i} \mathbf{v}_{l / c g}\right) & -S\left(\mathbf{J}_{i} \omega_{i}\right)
\end{array}\right]\left[\begin{array}{c}
i \mathbf{v}_{l / c g} \\
\omega_{i}
\end{array}\right]
$$

where $S(\cdot)$ is the cross-product operator. Note that centrifugal forces disappear in this formulation since the local reference frame is defined at the inertia center of the element, which is equivalent to having diagonal mass- and rotational inertia matrices.

\subsubsection{Gravity and buoyancy forces}

For an element with circular cross-section, the gravity and buoyancy forces are simply expressed as

$$
\mathbf{F}_{g b, i}={ }_{i} \mathbf{R}_{l / g}\left[0,0, \sigma_{i} \rho_{w} g L_{i} A_{i}-m_{i} g\right]^{\top}
$$

where $A_{i}=\frac{\pi}{4} d_{i}^{2}$ is the cross section area of element $i, d_{i}$ is the diameter of element $i, \rho_{w}$ is the density of water, $m_{i}$ is the dry mass of element $i, g$ is the acceleration of gravity and $\sigma_{i}$ is a coefficient expressing the percentage of submergence of the element,

$$
\sigma_{i}=\left\{\begin{array}{cl}
0 & \text { if }{ }_{i} z_{c g} \geq \mathbf{e}_{z}^{\top}{ }_{i} \mathbf{R}_{g / l} \mathbf{e}_{z} \frac{L_{i}}{2} \\
1 & \text { if } i z_{c g} \leq-\mathbf{e}_{z i}^{\top} \mathbf{R}_{g / l} \mathbf{e}_{z} \frac{L_{i}}{2} \\
-\frac{2 \cdot i z_{c g}-\mathbf{e}_{z i}^{\top} \mathbf{R}_{g / l} \mathbf{e}_{z} L_{i}}{2 \mathbf{e}_{z i}^{\top} \mathbf{R}_{g / l} \mathbf{e}_{z} L_{i}} & \text { otherwise }
\end{array}\right.
$$

where

$$
\mathbf{e}_{z}=[0,0,1]^{\top}
$$

\subsubsection{Hydrodynamic forces and added mass}

Slender structures are generally not affected by diffraction wave loads and viscous damping dominates. If the element is fully submerged and far from the surface the added mass may be considered as a constant addition to the rigid body mass. Constant added mass is included for the submerged part of an element since the acceleration of the element requires acceleration of the surrounding fluid. Submerged added mass is given as

$$
m_{a, i}=\sigma_{i} C_{a, i} \rho_{w} A_{i} L_{i}
$$

where $C_{a, i}$ is the added mass coefficient for element $i$ and $\sigma_{i}$ is as defined in Eq. (25). Note that the added mass is added to $\mathbf{M}_{i}$, e.g. $\mathbf{M}_{i}=\operatorname{diag}\left(\left(m_{i}+m_{a, i}\right)[1,1,1]\right)$ where $m_{i}$ is the dry-mass of element $i$, which also affects the Coriolis forces.

Viscous damping forces are here added as drag forces. The drag forces acting on a fully submerged lumped element $i$ due to an uniform current velocity may be expressed as

$$
\mathbf{F}_{\text {drag }, i}=-\left.\frac{1}{2} \rho_{w} \mathbf{S}_{i} \mathbf{C}_{d}\right|_{i} \mathbf{R}_{l / g} \mathbf{v}_{c}-\left.{ }_{i} \mathbf{v}_{l / c g}\right|^{\top}\left[{ }_{i} \mathbf{R}_{l / g} \mathbf{v}_{c}-{ }_{i} \mathbf{v}_{l / c g}\right]
$$

where $\mathbf{C}_{d}$ is a diagonal drag coefficient matrix, $\mathbf{S}_{i}=$ $\operatorname{diag}\left(\left[d_{i} L_{i}, d_{i} L_{i}, \pi d_{i} L_{i}\right]\right)$ is a diagonal matrix with the projected areas in the local body axes where $d_{i}$ is the element diameter. Note that if the element is not fully submerged, the conditions for the added mass simplification are violated and the flow characteristics around the element will differ from the conditions on which the drag coefficient is based. A simplified approach to calculate forces on semi-submerged cylinders can be implemented, where the drag is scaled with the percentage of submergence, e.g. similar to Eq. 25), and drag torques calculated due to the resultant drag force vector not acting in the center of gravity of the element. If the current experienced by an element is non-uniform the resultant drag force will not be in center of gravity, resulting in torques. Nevertheless, details regarding this will not be given more attention here.

The drag coefficients are usually calculated based on the Reynolds number and depend on the geometry and the surface roughness. One example is given in [24, p. 75] where drag forces on cables in near axial flow are studied. However, details regarding calculations of drag-and friction coefficients will not be treated here.

\subsubsection{Changing element lengths}

Another feature relevant for cables is changing the cable length. One way of doing this is to change the length of each lumped element. Since this changes the mass matrix, compensating forces are needed. Using Lagrange's equation

\footnotetext{
${ }^{\ddagger} C_{a, i} \approx 1$ for fully submerged slender cable elements in infinite fluid (far from any boundary conditions).
} 


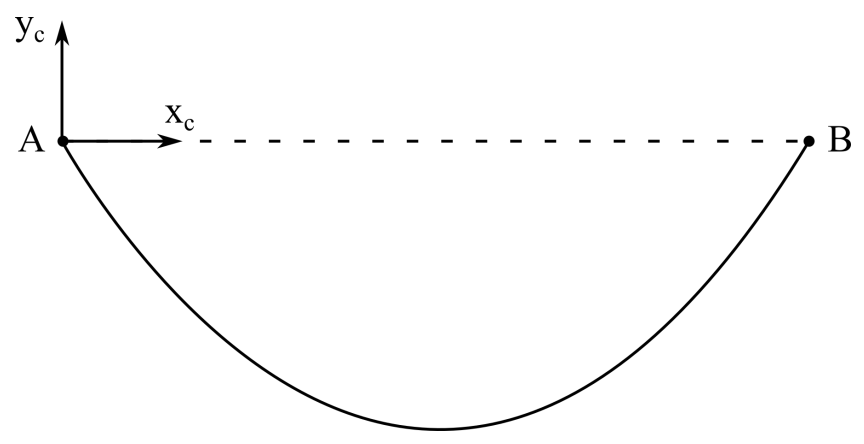

Fig. 3: Catenary calculations for initializing cable geometries, centered in a $2 \mathrm{D}$ view.

we can calculate these forces as

$$
\mathbf{F}_{\Delta L, i}=\left[\begin{array}{c}
-\rho_{i} A_{i} \dot{L}_{i} \cdot{ }_{i} \mathbf{v}_{l / c g} \\
-\frac{1}{16} \rho_{i} A_{i} d_{i}^{2} \dot{L}_{i} \omega_{i}-\frac{1}{4} A_{i} L_{i}^{2} \dot{L}_{i}\left[\begin{array}{lll}
1 & 0 & 0 \\
0 & 1 & 0 \\
0 & 0 & 0
\end{array}\right] \omega_{i}
\end{array}\right]
$$

where $\rho_{i}$ is the density of the cable material. Note that we also have to update $m_{i}, \mathbf{M}_{i}$ and $\mathbf{J}_{i}$ when an element length is changing. Also note that changing element lengths in a simulation might affect the numerical system stability due to changing the eigenvalues in the mass- and rotational inertia matrices.

\subsection{Initialization of cables}

Different strategies for initiating the geometry of cable systems exist. Here, a method for initializing the cable based on a catenary curve is presented. In particular, if a cable with at least two lumped elements has a length $L$ and is fixed between the points $\mathbf{p}_{A}$ and $\mathbf{p}_{B}$, assuming that $L>s$ where

$$
s=\sqrt{\left(\mathbf{p}_{B}-\mathbf{p}_{A}\right)^{\top}\left(\mathbf{p}_{B}-\mathbf{p}_{A}\right)}=\left\|\mathbf{p}_{B}-\mathbf{p}_{A}\right\|_{2}
$$

a catenary curve between the end points that has the desired cable length can be found by solving

$$
\min _{\delta \in \mathbb{R}_{>0}} f(\delta)=L-2 \delta \sinh \left(\frac{s}{2 \delta}\right)
$$

where $\delta$ is a tuning variable. When $\delta$ is found, the catenary curve is given as

$$
y_{c}=\delta \cosh \left(\frac{x_{c}-\frac{s}{2}}{\delta}\right)-\delta \cosh \left(\frac{s}{2 \delta}\right)
$$

where $y_{c}$ and $x_{c}$ are as defined in Fig. 3 When the catenary curve has been calculated the curve can be transferred to the global coordinate system and lumped elements placed on the curve, while also calculating the initial orientations for the lumped elements. Note that if the cable is too short, the length of the cable might be initialized in tension between the desired points. Also, if the cable only consists of one lumped element, the length of the cable is set equal to $s$ and the cable is initialized as a straight line between the desired end-points.

\subsection{Calculating tension and shear-forces}

The tension and shear forces, as well as the bending and torsion, are generated by the constraints and are calculated as

$$
\mathbf{F}_{c}=\Gamma\left(\dot{\mathbf{J H}} \mathbf{v}+\mathbf{J} \dot{\mathbf{H}} \mathbf{v}+2 \alpha \mathbf{J H} \mathbf{v}+\beta^{2} \mathbf{C}\right)
$$

Note that the term $\Gamma \mathbf{J H} \dot{\mathbf{v}}$, is neglected as it will be small compared to the other terms. One could also consider the same for the two first terms inside the parenthesis in Eq. (33). One reason for removing these smaller terms is that they contribute little to the overall behavior of the cable but introduce small numerical oscillations which we do not want to transmit as measurements to other systems, e.g. a tension control system for a winch. Also note that the constraint forces are given in the global reference frame and represents the net forces. Hence, the forces should be mapped to tension, shear forces, bending and torsion in local reference frames between each lumped element.

\section{Case studies}

The lumped cable models and the external point types have been implemented in a C++ library that also supports co-simulations through the Functional Mock-up Interface (FMI) standard [25]. Note that all derivative terms, such as $\mathbf{J}$ and $\frac{d}{d t} \mathbf{J}$ have been derived and implemented algebraically for all constraints presented in Sec. 2.4 in order to reduce numerical errors.

Before presenting a larger case related to in-sea seismic equipment, a basic case of two connected sinking cables is presented.

\subsection{Case 1: Sinking of connected cables}

In this case two cables are connected, as shown in Fig.4 where all cable ends are initially situated in the water surface. Note that the connection constraint is modeled as a ball-joint with no bending- or torsion stiffness. The outer ends of the cables, the end at point A and the end at point B in the figure, are connected to position controlled points, as discussed in Sec. 2.3.1 In this case, these points are keeps their respective initial positions. Both cables are equal in dimensions. Their main parameters are given in Table 2 For simplicity, the bending stiffness and the torsional stiffness are each set as a fraction of the axial stiffness. A linear current profile is calculated based on the surface and seabed currents (Table 2). The drag coefficients are calculated as functions of the 


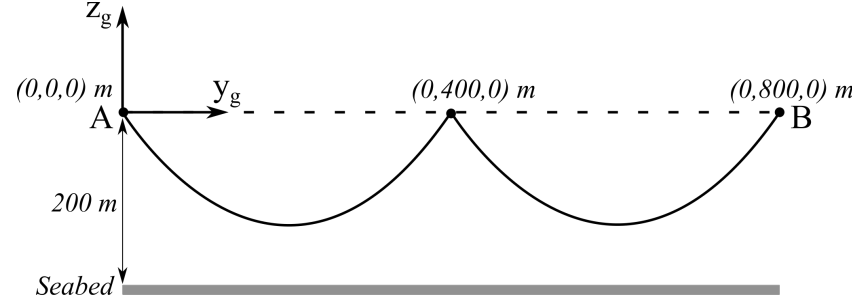

Fig. 4: Configuration of sinking connected cables

\begin{tabular}{|l|l|l|}
\hline Symbol & Description & Value \\
\hline$L$ & Cable length & $500 \mathrm{~m}$ \\
$n$ & Number of elements in each cable & 50 \\
$d$ & Cable diameter & $0.044 \mathrm{~m}$ \\
$\rho$ & Cable density & $1100 \mathrm{~kg} / \mathrm{m}^{3}$ \\
$\rho_{w}$ & Water density & $1025 \mathrm{~kg} / \mathrm{m}^{3}$ \\
$E$ & Elasticity modulus & $120 \mathrm{GPa}$ \\
$\alpha$ & Baumgarte stabilization damping & 50 \\
$\varepsilon$ & Elastic Baumgarte parameter & $10^{-8}$ \\
$\frac{E I}{E A}$ & Fraction of bending- and axial stiffness & 0.01 \\
$\frac{G J}{E A}$ & Fraction of torsion- and axial stiffness & 0.01 \\
$z_{\text {depth }}$ & Depth & $-200 \mathrm{~m}$ \\
$\mathbf{v}_{c, 1}$ & Current 1 at surface & $(0.5,0,0) \mathrm{m} / \mathrm{s}$ \\
$\mathbf{v}_{c, 2}$ & Current 2 at seabed & $(0.0,0,0) \mathrm{m} / \mathrm{s}$ \\
\hline
\end{tabular}

Table 2: Main parameters in Case 1: Sinking connected cables.

Reynolds number. Seabed contact forces are implemented as depth constraints on each element.

The total cable system consists of $1328 \$$ states. The total system is simulated as one model using the explicit Euler integration method with a time-step size of $0.0125 \mathrm{~s}$. To reach steady state, it is simulated for $1000 \mathrm{~s}$ (simulation time).

The system was compiled as a standalone executable and the simulation finished after approximately $195 \leq$ making it about 5.1 times faster than real-time. A graphical representation of the cable system geometry throughout the simulation made using the Mayavi library available in Python. The results for the 500 first seconds, which is approximately the time it takes for the cables to sink to the bottom, are shown in Fig. 5 .

The figure shows that the cable system follows the current profile direction with the progression of simulation time. The two cables touch the seafloor at approximately $300 \mathrm{~s}$ (Fig. 5d) and both cables start to land towards each other. After approximately $500 \mathrm{~s}$ (Fig. [5f), the entire cable system has come to rest and a small buckle in the connection of the two cables can be seen. This is expected since the cables

\footnotetext{
${ }^{\S}$ There are 13 states in each lumped cable element and in each point type, and there is 1 additional state in each cable related to the winching functionality. Hence, 102 elements $\times 13$ states per element $+2=1328$.

IUsing a normal workstation laptop, HP ZBook 15 G4, late 2017 mod.
}

\begin{tabular}{|l|l|l|}
\hline Symbol & Description & Value \\
\hline$\alpha$ & Baumgarte stab. damping & {$[1,5,10,20,30,50]$} \\
$\varepsilon$ & Elastic Baumgarte parameter & {$\left[10^{-7}, 10^{-8}, 10^{-9}, 10^{-10}\right]$} \\
- & Numerical solver & Variable Runge-kutta 2 \\
- & Relative tolerance & $10^{-5}$ \\
- & Absolute tolerance & $10^{-5}$ \\
- & Simulation time & $50 \mathrm{~s}$ \\
\hline
\end{tabular}

Table 3: Main parameters in efficiency study.

land towards each other at the seabed and when there is not enough space for the cables to completely extend, the remaining falls to the side, as is shown in the figure. The size of the buckle that falls to the side is also determined by the bending stiffness of the cables.

\subsubsection{Efficiency study}

The Baumgarte stabilization method has been thoroughly studied, validated, and verified already in the literature, the same yields the environmental loads and friction characteristics presented in Sec. 2. What is new in the proposed method is the introduction of elasticity in the Baumgarte stabilization, which is crucial for real-time applications where a singularity in a matrix inversion can cause a crash in the application, in the best case produce non-valued results. To further study the effectiveness of the proposed cable modeling method, a small study with varying $\varepsilon$ and $\alpha$ is performed. In this study we lift the cable arrangement out of water and simulate its response using a variable time-step size solver. The new parameters not previously listed in Table 2 are found in Table 3 .

Fig. 6 shows a comparison of the simulation results. Fig. 6a and Fig. 6b show how the choice of $\alpha$ and $\varepsilon$ affects simulation time and error, respectively. Since the choice of parameters in this case affects the dynamics of the system, and not the solver, the definition of error is not straightforward. A simulation which is 'well behaved' in simulation performance and accuracy is selected as the reference and the error is then the difference between the reference and current simulation. Fig. 6a indicates that the simulation time increases with increased $\varepsilon$, and particularly when $\alpha$ is low. This is expected since less damping of the constraints, and more flexibility in the constraint formulations, give room for more numerical oscillations. The reason for high values of $\varepsilon$ giving longer simulation times for low values of $\alpha$ is mixed. Lowering $\varepsilon$ will result in a filtering effect of the cable dynamics and the corresponding constraints. Since the diagonal of the mass matrix becomes more significant, the matrix inversion algorithm will converge faster and become more flexible with respect to numerical errors. This lowers the simulation time and is also why increasing $\alpha$ for low values of $\varepsilon$ have less effect on the total simulation time. This filtering effect is illustrated in Fig. 7, where two different values for $\varepsilon$ are compared visually. But it is not possible to see the oscil- 


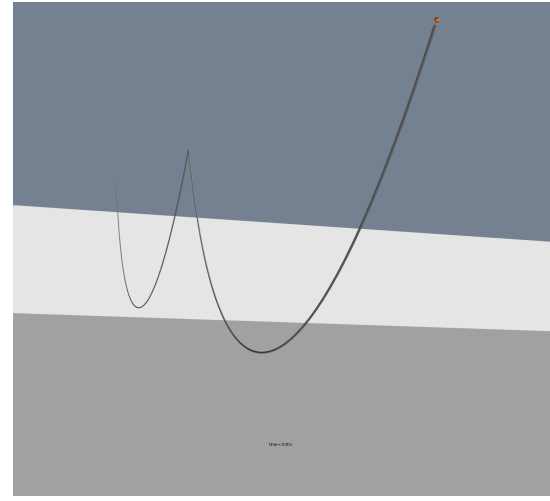

(a) time $\approx 0 \mathrm{~s}$

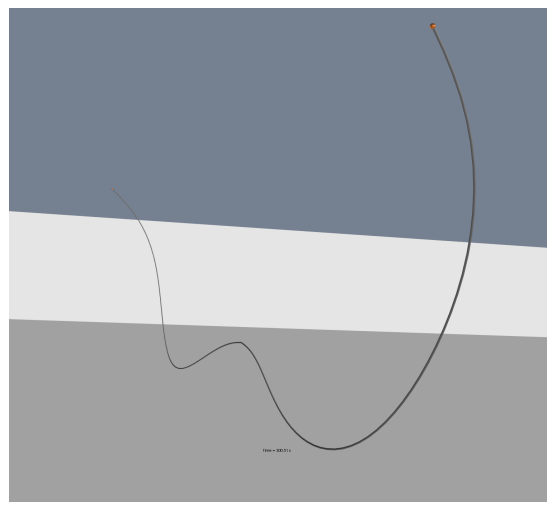

(d) time $\approx 300 \mathrm{~s}$

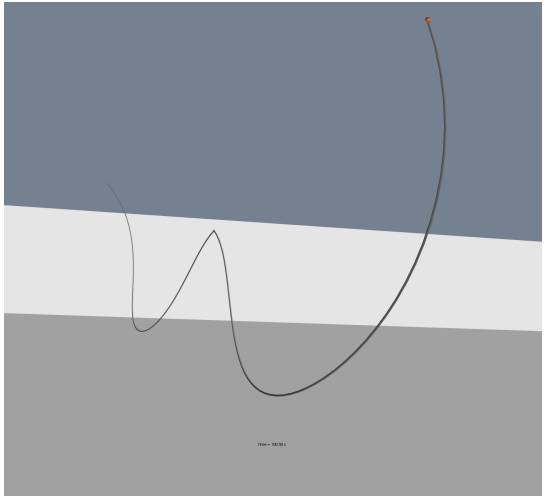

(b) time $\approx 100 \mathrm{~s}$

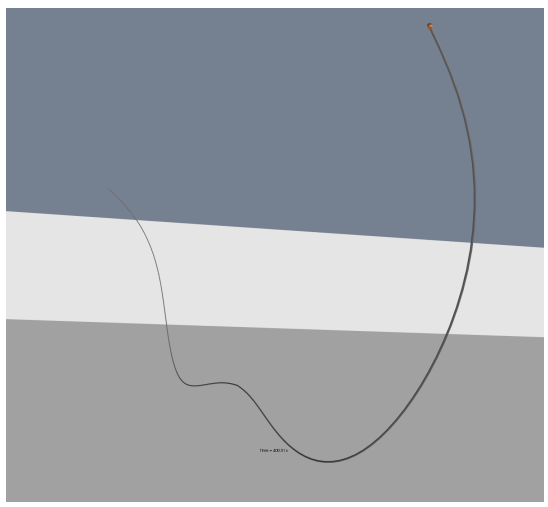

(e) time $\approx 400 \mathrm{~s}$

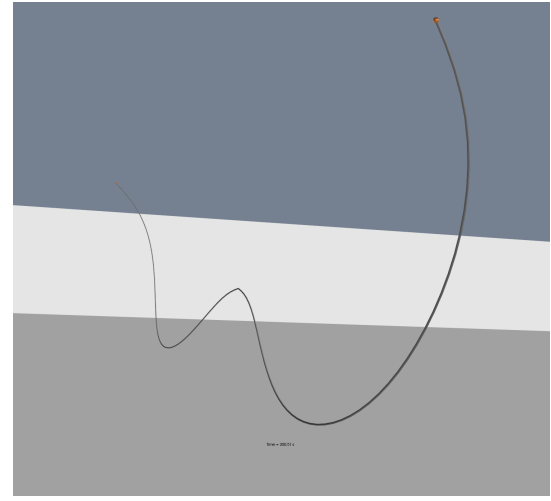

(c) time $\approx 200 \mathrm{~s}$

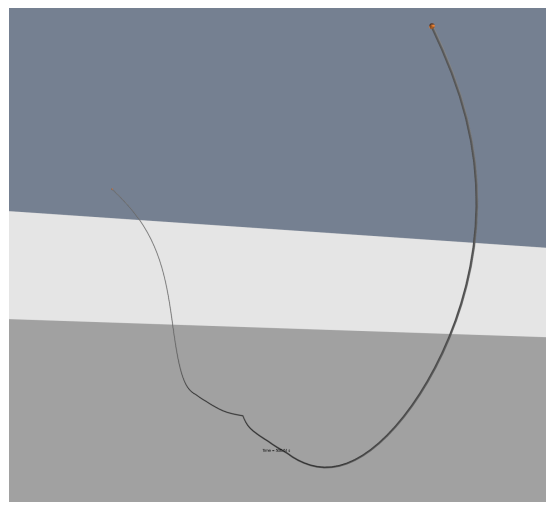

(f) time $\approx 500 \mathrm{~s}$

Fig. 5: Graphical representation of the simulation results from the sinking connected cable example. Note that the blue color in the figure represents the water surface while the gray color, not being the cables, represents the seafloor. The fixed end positions are represented by orange spherical elements in the figure.

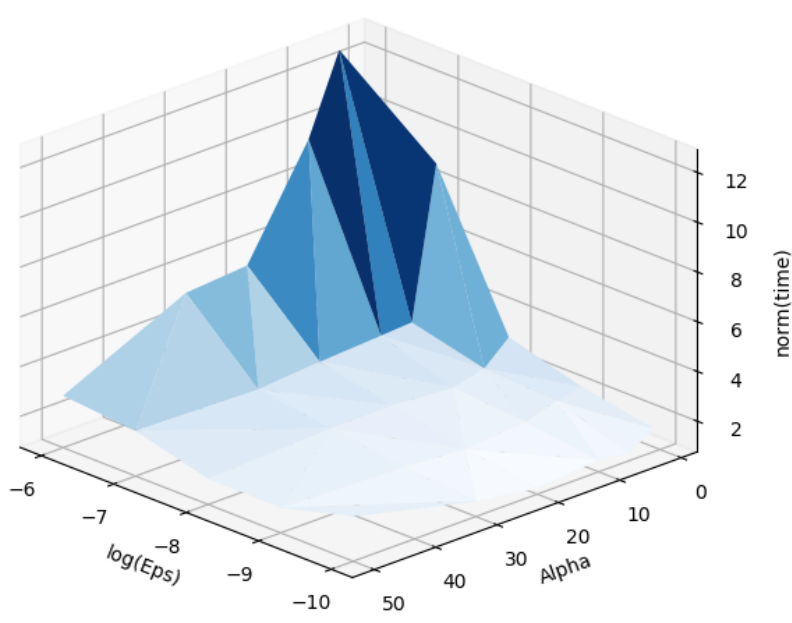

(a) Normalized total simulation time.

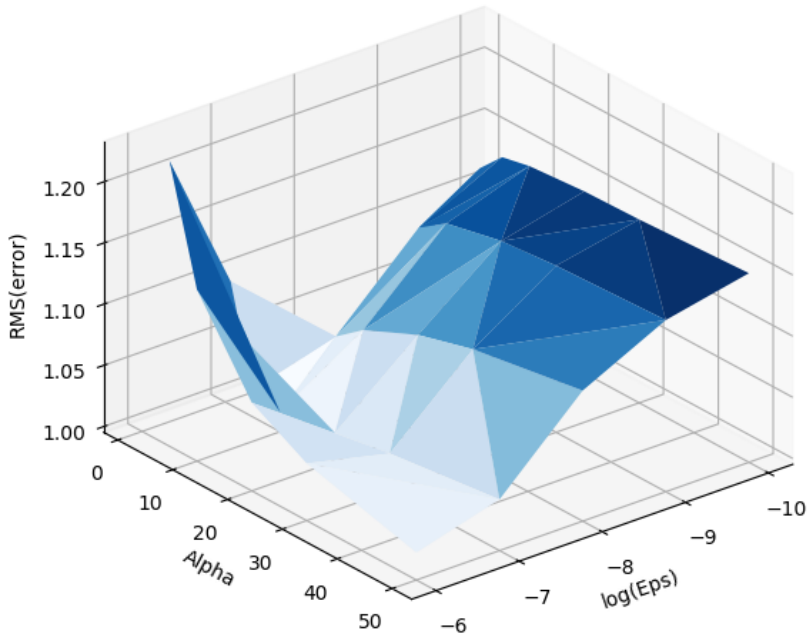

(b) RMS state vector error, RMS(error) $=\log _{10}(\sigma($ error $)+10)$.

Fig. 6: Comparison of results when varying $\varepsilon$ and $\alpha$. Note that the orientation of the plots are not equal.

lations along the cables with the highest value of $\varepsilon$ in the figure. These oscillations are triggered by the cable being dropped in a gravity field without any significant damping. The case with the lowest value of $\varepsilon$, however, drops down and reach an equilibrium point almost momentarily without experiencing any oscillations, as if critically over-damped.
High values of $\varepsilon$, on the other hand, makes the constraints more flexible. This can give rise to more numerical vibrations in the cable arrangement, which causes the solver to use smaller time-step sizes to meet the solver tolerances. Except for the lowest values of $\varepsilon$, increasing values of $\alpha$ gives increased time-step sizes. This is caused by increased con- 


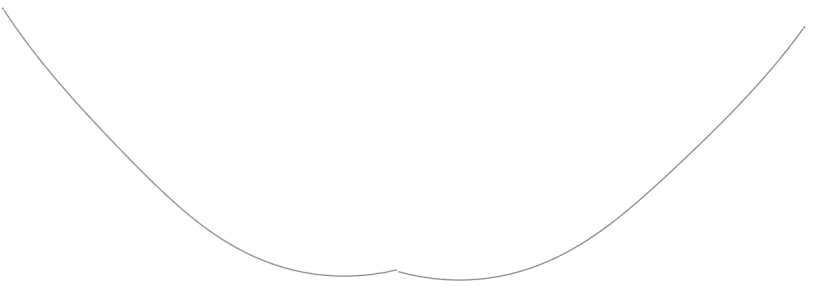

(a) $\varepsilon=10^{-12}, \alpha=1.0$

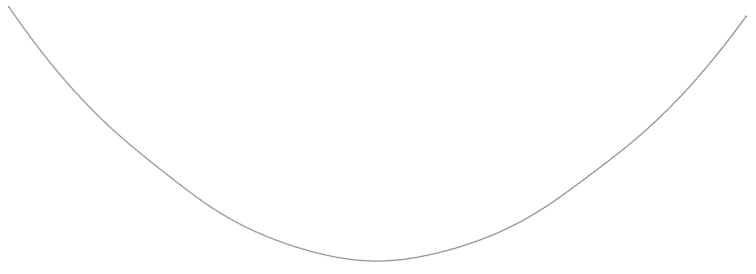

(b) $\varepsilon=10^{-8}, \alpha=1.0$

Fig. 7: Filtering effect on cable dynamics from low values of $\varepsilon$, where the intended dynamics in the connection between the cables are violated with low values of $\varepsilon$. Note that the connection point between the two cables are not subject to bending stiffness, which is the case for the cable elements within each cable. Also note that the cable in Fig. $7 \mathrm{~b}$ has not reached its steady state at this point, it has oscillations along the cable caused by being dropped in a gravity field without significant external damping.

straint damping. Experience indicate that when using a fixed step-size solver, such as the Euler integration method, higher values of $\alpha$ tend to stabilize the solver, allowing higher fixed time-step sizes. On the other hand, lower values of $\varepsilon$ tend to destabilize the solver. Hence, real-time applications using fixed step size solvers will probably benefit from higher values of $\varepsilon$ to relax the system and increase the time-step size.

Fig. $6 \mathrm{~b}$ shows the RMS error of the state vector when using a simulation with $\varepsilon=10^{-8}$ and $\alpha=1.0$ as reference. $\varepsilon=10^{-8}$ is chosen to avoid the possible filtering effect lower values of $\varepsilon$ have on the simulation results. The constraint damping can be related to material damping properties. This means that the best choice of $\alpha$ is highly case dependent, and can not be chosen solely based on $\varepsilon$. The correlation between the values of $\varepsilon$ and $\alpha$ with the deviation from the reference simulation and the normalized total simulation time is seen in the figure indicates that there is a possibility of reducing simulation fidelity to gain simulation speed while maintaining acceptable results compared to the reference. However, it is advised to check the resulting dynamics in systematically in order to verify that the resulting dynamics maintains the global system response characteristics.

\subsection{Case 2: A seismic front end}

Seismic streamer cables are used in the search of new offshore oil and gas resources. Seismic cable spreads consist of up to twenty $10 \mathrm{~km}$ cables towed behind a vessel, spanning an area of up to $18 \mathrm{~km}^{2}$, making them the largest man-made moving objects on Earth. A seismic front end, as shown in Fig. 8, is the cable arrangement in front of the seismic streamer cables. Its purpose is both to distribute the towing loads and to keep the seismic cables separated. In the system shown in the figure, the lead-in cables distribute the load of the entire towing operation of the seismic in-sea equipment. The deflectors keep the spread separated, and the spread ropes keep a more or less constant distance between the head of the streamer cables. The spur-lines connect the deflectors to the streamers, while the wide-tow cables connect the deflectors to the vessel itself. In addition to the cables, deflectors and buoys, a gun-array is often situated in

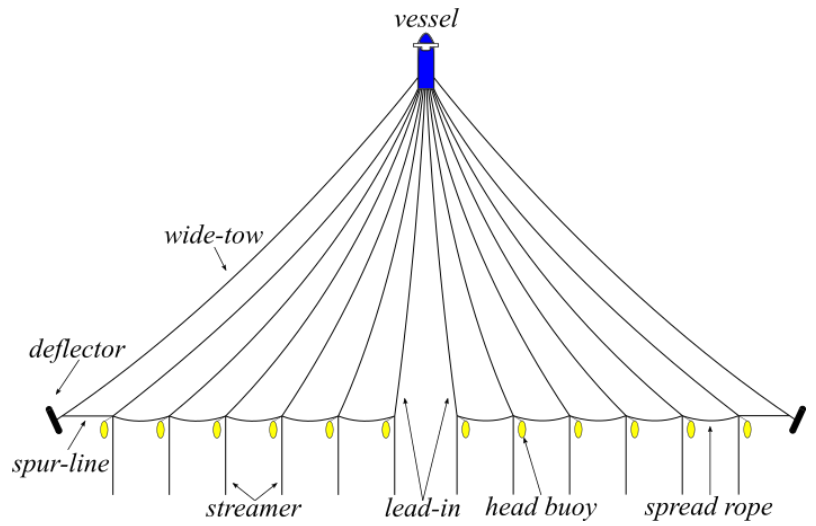

Fig. 8: Sketch of a seismic front end [22].

the center of the front end. The gun-array is a larger buoy centered between the inner lead-in cables with air-guns submerged below it. The air-guns are used to produce the sound waves for recording the seismic data. The gun-array itself is not part of the seismic front end, but may be connected to it, as explained shortly.

Some information about the condition of the front end is available for the seismic vessel crew during normal operations. The winch systems may provide tension measurements and cable length estimates, but these are often of poor quality. RGPS\| on the head buoys can provide position references for these. Measurements of cable orientations are sometimes available for some points along the cable, as well as depth measurements. Still, operational decisions are highly based on experience and best practice. A state observer for the front end of a seismic spread was therefore developed in the industry research project Real-time digital twin for boosting performance of seismic operations.

In this case study, the front end state observer model is divided into two parts; a port-side part and a starboard part. Each side consists of 8 lead-in cables, one wide-tow cable, one spur-line cable, 7 separation rope cables, 8 buoy cables, one sliding cable connecting the gun-array to a lead-

${ }^{\|}$Relative GPS 


\begin{tabular}{|c|c|c|}
\hline Cable description & Connections & Main parameters \\
\hline $\begin{array}{l}\text { Lead-in cable } i \in \\
\{1,8\}\end{array}$ & $\begin{array}{l}\text { Start: Lead-in } \\
\text { winch } i \\
\text { End: End of buoy } \\
\text { cable } i\end{array}$ & $\begin{array}{l}\text { Number of ele- } \\
\text { ments: } 20 \\
\text { Diameter: } 0.044 \mathrm{~m} \\
\text { Density: } 1110 \mathrm{~kg} / \mathrm{m}^{3} \\
\text { Length: } 550-925 \mathrm{~m}\end{array}$ \\
\hline $\begin{array}{ll}\text { Buoy } & \text { cable } \\
i \in\{1,8\} & \end{array}$ & $\begin{array}{l}\text { Start: Buoy } i \\
\text { End: Below buoy } i\end{array}$ & $\begin{array}{l}\text { Number of ele- } \\
\text { ments: } 1 \\
\text { Diameter: } 0.044 \mathrm{~m} \\
\text { Density: } 1110 \mathrm{~kg} / \mathrm{m}^{3} \\
\text { Length: } 50 \mathrm{~m}\end{array}$ \\
\hline $\begin{array}{l}\text { Separation rope ca- } \\
\text { ble } i \in\{1,7\}\end{array}$ & $\begin{array}{l}\text { Start: End of buoy } \\
\text { cable } i \\
\text { End: End of buoy } \\
\text { cable } i+1\end{array}$ & $\begin{array}{l}\text { Number of ele- } \\
\text { ments: } 10 \\
\text { Diameter: } 0.044 \mathrm{~m} \\
\text { Density: } 1110 \mathrm{~kg} / \mathrm{m}^{3} \\
\text { Length: } 110 \mathrm{~m}\end{array}$ \\
\hline Spur-line cable & $\begin{array}{l}\text { Start: End of buoy } \\
\text { cable } 7 \\
\text { End: Deflector }\end{array}$ & $\begin{array}{l}\text { Number of ele- } \\
\text { ments: } 10 \\
\text { Diameter: } 0.044 \mathrm{~m} \\
\text { Density: } 1110 \mathrm{~kg} / \mathrm{m}^{3} \\
\text { Length: } 200 \mathrm{~m}\end{array}$ \\
\hline Wide-tow cable & $\begin{array}{l}\text { Start: Wide-tow } \\
\text { winch } \\
\text { End: Deflector }\end{array}$ & $\begin{array}{l}\text { Number of ele- } \\
\text { ments: } 20 \\
\text { Diameter: } 0.044 \mathrm{~m} \\
\text { Density: } 1110 \mathrm{~kg} / \mathrm{m}^{3} \\
\text { Length: } 1100 \mathrm{~m}\end{array}$ \\
\hline Gun-array cable & $\begin{array}{l}\text { Start: Lead-in ca- } \\
\text { ble 2, initially at the } \\
\text { middle } \\
\text { End: Gun-array }\end{array}$ & $\begin{array}{l}\text { Number of ele- } \\
\text { ments: } 10 \\
\text { Diameter: } 0.044 \mathrm{~m} \\
\text { Density: } 1110 \mathrm{~kg} / \mathrm{m}^{3} \\
\text { Length: } 110 \mathrm{~m}\end{array}$ \\
\hline
\end{tabular}

Table 4: Cable configuration in each side of the front end.

in cable and 19 external position inputs. Table 4 summarizes the configuration of the cables in each side of the front end. Note that the gun-array cables are connected to the lead-in cables through a sliding constraint, as discussed in Sec. 2.4

In the observer, the cables are fed with live position measurements from the buoys, the deflectors and the winchpositions through position controlled points, as shown in Fig. 9, and the output from the state observer is the front end geometry and tensions for the various cables and ropes. The initial positions of the "points" in the system are shown in Table 5 Note that the position $(0,0,0) \mathrm{m}$ is the center of gravity for the vessel, and that the positions are set relative to the vessel. The main cable- and environmental parameters are listed in Table 6 In this case, the current is set to zero and the vessel speed is set to $1.5 \mathrm{~m} / \mathrm{s}$ ( $x_{g}$-direction).

To excite the front end observer, we add noise to the virtual RGPS measured $y$-positions of the buoys and the deflectors after $100 \mathrm{~s}$ (simulation time). This noise is implemented as a sine-function with amplitude $50 \mathrm{~m}$ and frequency $0.01 \mathrm{rad} / \mathrm{s}$. A load of $10 \mathrm{kN}$ is added to the end of each lead-in cable, in the $x_{g}$-direction, to simulate streamer cable loads. In a realistic application these loads would be timevarying, and measurements of the resulting tension would be provided as input to the state observer.

The total state-space model for each side of the front end consists of 3887 states. An explicit Euler integration method is used in the state observer, with time-step size $0.025 \mathrm{~s}$. Co-

\begin{tabular}{|l|l|l|}
\hline Point & $\begin{array}{l}\text { Port-side positions } \\
(x, y, z)[\mathrm{m}]\end{array}$ & $\begin{array}{l}\text { Starboard posi- } \\
\text { tions }(x, y, z)[\mathrm{m}]\end{array}$ \\
\hline Buoy 1 & $(-550,50,0)$ & $(-550,-50,0)$ \\
\hline Lead-in winch 1 & $(0,1,10)$ & $(0,-1,10)$ \\
\hline Buoy 2 & $(-550,150,0)$ & $(-550,-150,0)$ \\
\hline Lead-in winch 2 & $(0,2,10)$ & $(0,-2,10)$ \\
\hline Buoy 3 & $(-550,250,0)$ & $(-550,-250,0)$ \\
\hline Lead-in winch 3 & $(0,3,10)$ & $(0,-3,10)$ \\
\hline Buoy 4 & $(-550,350,0)$ & $(-550,-350,0)$ \\
\hline Lead-in winch 4 & $(0,4,10)$ & $(0,-4,10)$ \\
\hline Buoy 5 & $(-550,450,0)$ & $(-550,-450,0)$ \\
\hline Lead-in winch 5 & $(0,5,10)$ & $(0,-5,10)$ \\
\hline Buoy 6 & $(-550,550,0)$ & $(-550,-550,0)$ \\
\hline Lead-in winch 6 & $(0,6,10)$ & $(0,-6,10)$ \\
\hline Buoy 7 & $(-550,650,0)$ & $(-550,-650,0)$ \\
\hline Lead-in winch 7 & $(0,7,10)$ & $(0,-7,10)$ \\
\hline Buoy 8 & $(-550,750,0)$ & $(-550,-750,0)$ \\
\hline Lead-in winch 8 & $(0,8,10)$ & $(0,-8,10)$ \\
\hline Deflector 8 & $(-550,900,-1)$ & $(-550,-900,-1)$ \\
\hline Wide-tow winch 8 & $(0,10,10)$ & $(0,-10,10)$ \\
\hline Gun-array 8 & $(-250,0,-50)$ & $(-250,0,-50)$ \\
\hline
\end{tabular}

Table 5: Initial positions. These positions are given relative to the vessel.

\begin{tabular}{|c|c|c|c|}
\hline & Parameter & Description & Value \\
\hline \multirow[t]{4}{*}{ 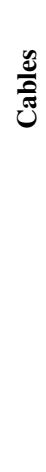 } & $\alpha$ & $\begin{array}{l}\text { Damping parame- } \\
\text { ter in the Baum- } \\
\text { garte stabilization } \\
\text { method }\end{array}$ & 10 \\
\hline & $\mu$ & $\begin{array}{l}\text { Sliding friction } \\
\text { parameter }\end{array}$ & 0.1 \\
\hline & $\frac{E I}{E A}=\frac{G J}{E A}$ & $\begin{array}{l}\text { Ratio between } \\
\text { bending stiffness } \\
\text { / torsion stiffness } \\
\text { and axial stiffness }\end{array}$ & 0.05 \\
\hline & $\begin{array}{l}\text { Streamer load- } \\
\text { cells }\end{array}$ & - & $\begin{array}{l}-10 \mathrm{kN} \text { in global } x \text { - } \\
\text { direction }\end{array}$ \\
\hline \multirow{5}{*}{ } & $\rho_{w}$ & Water density & $1025 \mathrm{~kg} / \mathrm{m}^{3}$ \\
\hline & Water temperature & - & $12^{\circ}$ \\
\hline & Current 1 & At $0 \mathrm{~m}$ depth & $\begin{array}{l}(0,0) \quad \mathrm{m} / \mathrm{s}, \quad \text { global } \\
x_{g}, y_{g} \text {-direction }\end{array}$ \\
\hline & Current 2 & At $10 \mathrm{~m}$ depth & $\begin{array}{l}(0,0) \quad \mathrm{m} / \mathrm{s}, \quad \text { global } \\
x_{g}, y_{g} \text {-direction }\end{array}$ \\
\hline & Vessel speed & $x_{g}$-direction & $1.5 \mathrm{~m} / \mathrm{s}$ \\
\hline
\end{tabular}

Table 6: Main cable- and environmental parameters. 


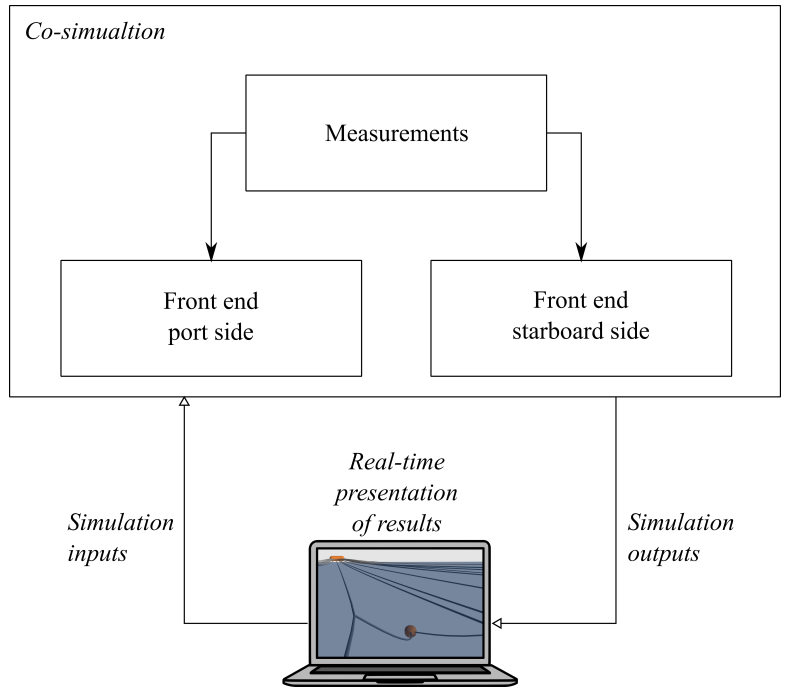

Fig. 9: Co-simulation setup of the seismic front end observer.

simulation is employed, enabling us to simulate each side of the front end in a different integrator. The co-simulation macro time-step size is set to $0.25 \mathrm{~s}$, and the simulation is run for $2500 \mathrm{~s}$ (simulation time). A separate co-simulation module is used for generating the virtual RGPS measurement.

The position changes are fed to the state observer cosimulation models using a third co-simulation model used for generating the RGPS measurements. The entire simulation was performed on a quite standard laptor**, using the Coral co-simulation master algorithm [26]. The simulation run about 3 times faster than real-time.

The observed geometry of the front end, as seen from the side, is shown in Fig. 10 for two different simulation times. This is created by the Mayavi Python library. Detailed results and their discussion is outside the scope of this paper. After a while, before we start to excite the model with buoy position changes, the cables begin to converge to their corresponding steady-state positions, as shown in Fig. $10 \mathrm{~b}$ However, as the results show, about 100 seconds is not enough for the widetow cables to converge to their steady-state positions.

The forces between the two middle lead-in cables and their winches when changing the buoy and deflector positions are shown in Fig. 11, while the geometry of the front end is shown in Fig. 12, By comparing Fig. 11a and Fig. 11b we see that the shear forces in $x$ and the tension forces are in opposite phases, as expected since the front end is mirrored about the $x_{g}$-axis and since both models share the same forces and the same environmental conditions. Nevertheless, the results show that the tension forces varies from about $13.3 \mathrm{kN}$ to about $14.5 \mathrm{kN}$. These tension variations come as a result of adding the cyclic variations in RGPS position changes for the head buoy and the deflectors. The effect of these variations are shown in detail in Fig. 12, which shows the neutral front end geometry in Fig. 12a, the maximal portside deflection of the front end in Fig. 12b and the maximal starboard deflection of the front end in Fig. 12c Note that

\footnotetext{
${ }^{* *}$ HP ZBook 15 G4, late 2017 mod.
}

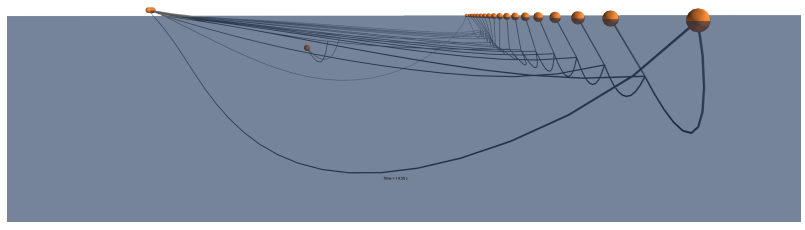

(a) Front end side-view right after simulation start.

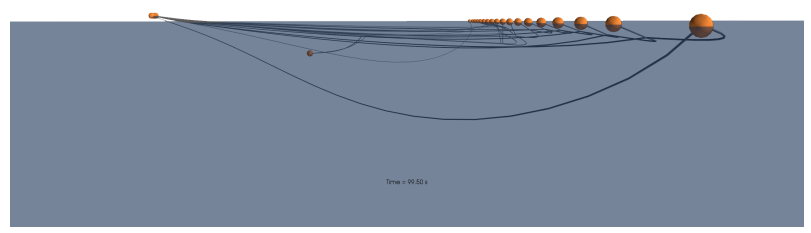

(b) Front end side-view right before changing buoy and deflector positions.

Fig. 10: Side views of the seismic front end geometry.

the lead-in and wide-tow cables get slack when the buoy positions move towards the center line. The reason is that as we only change the $y$-position, the distances between the buoys and the winches are not kept constant.

As a minor validation of the simulated cable tensions in this case an analytic solutions of a suspended cable having a catenary geometry was used for comparison. The results from the analytic solution coincide with the simulation results.

\section{Conclusion}

This article presents a robust, real-time capable lumped modeling strategy for flexible, slender marine systems. Constraints are used for modeling the connections between the lumped elements, in addition to the effects of some external forces. The constraint types presented in this article are position constraints, bending- and torsion constraints, sliding end constraint involving friction, and depth constraints. The resulting vector of constraints are transformed to explicit constraint forces by using an elastic version of Baumgarte stabilization. The constraint force dynamics are tuned to match material properties and system characteristics, such as axial stiffness and friction coefficients. Different environmental forces that may act on the lumped models are presented. This includes buoyancy, gravity and hydrodynamic drag forces. In addition, forces related to winching functionality, which changes the length of each lumped element, and forces related to rotating coordinate frames are included. A procedure to estimate a catenary curve between two points 

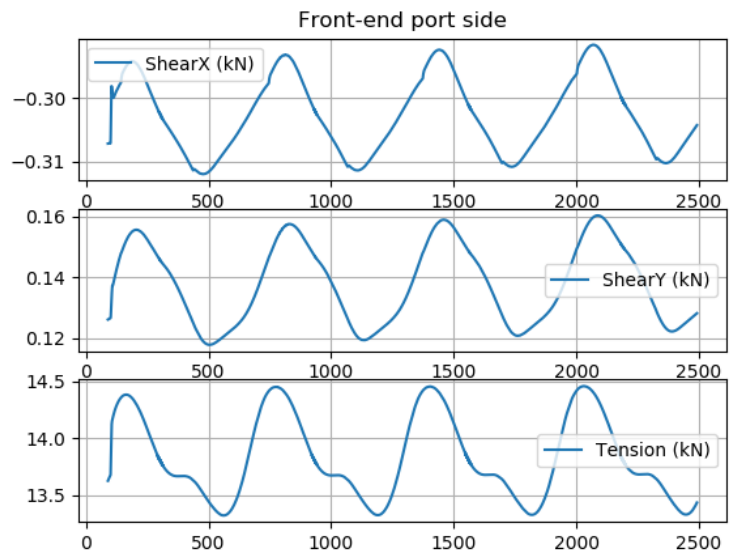

(a) Forces in port-side lead-in cable 1, at the winch connection point.
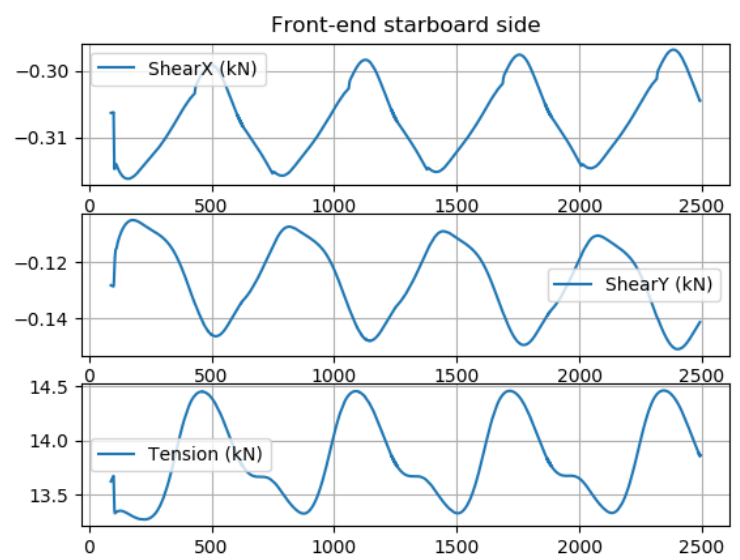

(b) Forces in starboard lead-in cable 1, at the winch connection point.

Fig. 11: Forces in the center lead-in cables for simulation time $t \in[100,2500]$.

are presented. This method is used to initialize cable geometries if the cable itself is longer than the distance between the initial cable end points.

The proposed modeling approach was implemented as a C++ library, facilitating the modeling of flexible, slender marine structures. Two case studies were presented. In the first case study, two connected cables were attached between two fixed points. The simulation started in the water surface, and a linear current profile was used. The simulation showed the sinking process until the cables found their steady state, where parts of the cables were suspended from the fixed points and parts were resting on the seabed. Also, a short efficiency study regarding variations in the parameters $\varepsilon$ and $\alpha$ were conducted. In general, the results indicate that it is possible to reduce accuracy in intended model dynamics at the same time as increasing the simulation speed. This while assuring safe matrix inversions without any singularities. This is one of the benefits of the method and is considered useful when dealing with large cable systems in real-time applications, which in general require high computational efforts. Allowing such a property also comes with a prices, namely that care must be take when choosing the values of $\alpha$ and $\varepsilon$ such that the cable system dynamics remains as intended.

In the second case study, a marine seismic front end was simulated. Using co-simulation, the simulation was divided into three parts: The port front end, the starboard front end and the measurements from the buoys and deflectors.

The case studies gave some insights to the real-time capabilities of the implemented cable library. Case study 1 and 2 consisted of 1328 and 3887 states, respectively. Stable simulations were achieved in both cases, and they were able to simulate 5 and 3 times faster than real-time, respectively.

\section{Acknowledgements}

The authors would like to thank the industry research project Real-time digital twin for boosting performance of seismic operations (RCN project number 282378) for providing the main case study of the seismic front end state observer. The authors would like also to thank Jørgen Haavind Jensen for early work on development of the Elastic Baumgarte method.

\section{References}

[1] Sævik, S., and Gjosteen, J. K., 2012. "Strength analysis modelling of flexible umbilical members for marine structures". Journal of Applied Mathematics.

[2] Karnopp, D. C., Margolis, D. L., and Rosenberg, R. C., 2006. System Dynamics: Modeling and Simulation of Mechatronic Systems. John Wiley \& Sons, Inc., New York, NY, USA.

[3] Pedersen, E., 2009. "Rotordynamics and bond graphs: basic models". Mathematical and Computer Modelling of Dynamical Systems, 15(4), pp. 337-352.

[4] Torsvik, J., and Pedersen, E., 2020. "On the modeling of rotors with rolling element bearings using bond graphs". Journal of Sound and Vibration, 473.

[5] Yang, L., Hals, J., and Moan, T., 2012. "Comparative study of bond graph models for hydraulic transmission lines with transient flow dynamics". Journal of Dynamic Systems, Measurement and Control, Transactions of the ASME, 134(3), pp. 1-13.

[6] Karnopp, D., 1975. "Lumped parameter models of acoustic filters using normal modes and bond graphs". Journal of Sound and Vibration.

[7] Lee, C.-W., Kim, Y.-B., Lee, G.-H., Choe, M.-Y., Lee, M.-K., and Koo, K.-Y., 2008. "Dynamic simulation of a fish cage system subjected to currents and waves". Ocean Engineering, 35(14-15), pp. 1521-1532.

[8] Reite, K.-J., Føre, M., Aarsæther, K. G., Jensen, J., Rundtop, P., Kyllingstad, L. T., Endresen, P. C., Kristiansen, D., Johansen, V., and Fredheim, A., 2014. "Fhsim-time domain simulation of marine systems". In ASME 2014 33rd International Conference on Ocean, Offshore and Arctic Engineering, 
(a) Front end overview right before changing buoy and deflector positions.

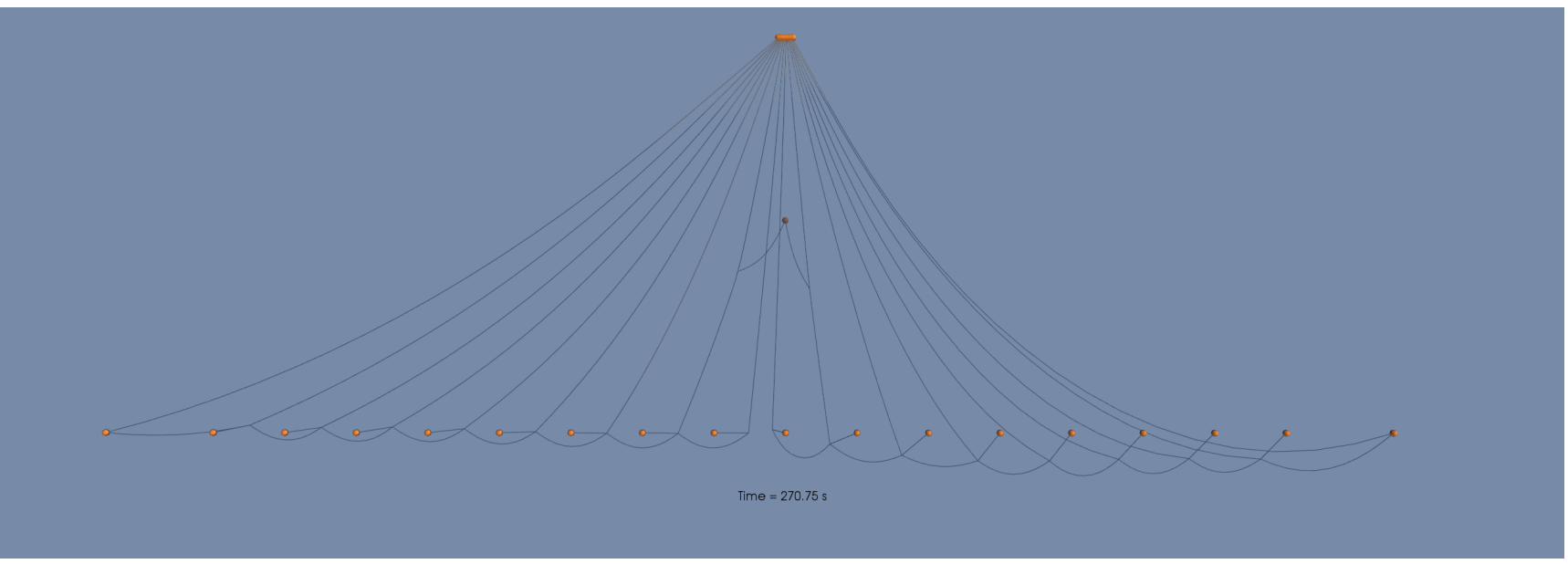

(b) Front end overview when buoys and deflector have maximal $y$-position values.

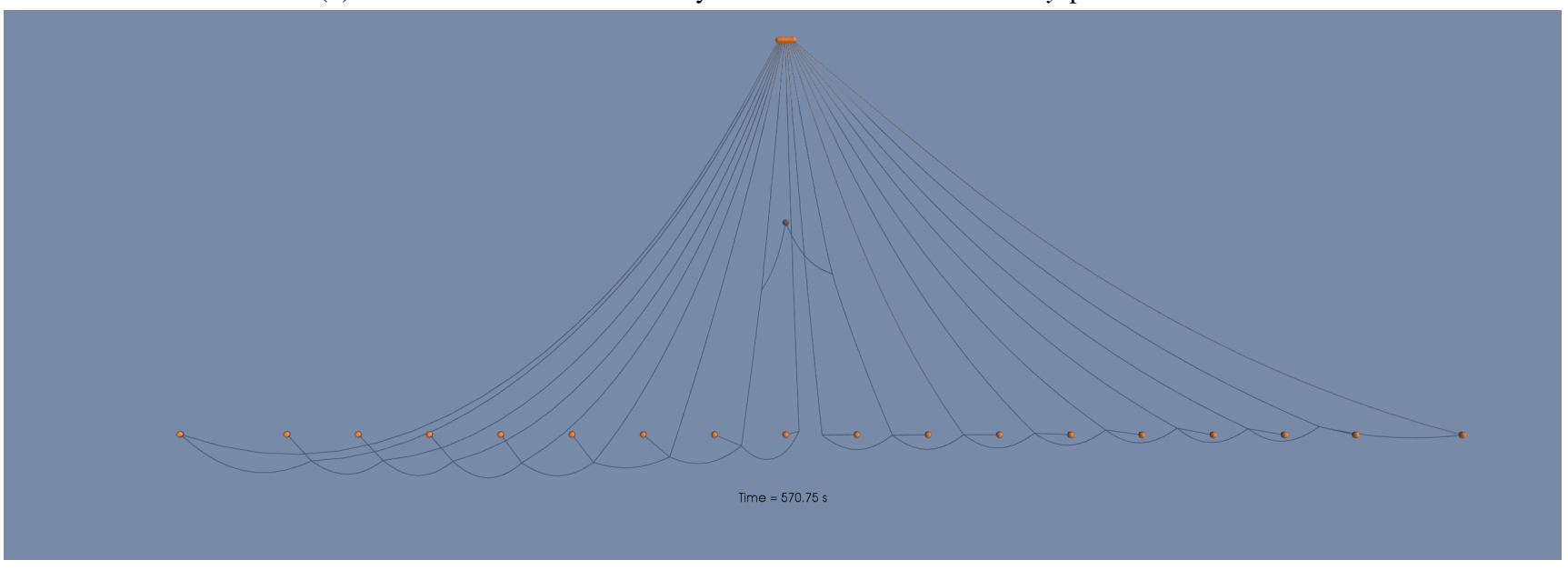

(c) Front end overview when buoys and deflector have minimal $y$-position values.

Fig. 12: Overview of front end geometry.

Vol. 8A, American Society of Mechanical Engineers, pp. V08AT06A014-V08AT06A014.

[9] Reite, K.-J., 2009. "State estimator for trawl system surveillance". In Control. Eng. Fish, pp. 179-186.

[10] Moe-Føre, H., Endresen, P., Aarsæther, K., Jensen, J., Føre, M., Kristiansen, D., Fredheim, A., Lader, P., and
Reite, K.-J., 2014. "Structural analysis of aquaculture nets: Comparison and validation of different numerical modelling approaches". In Proceedings of the International Conference on Offshore Mechanics and Arctic Engineering - OMAE, Vol. 7.

[11] Pedersen, E., and Pedersen, T. A., 2005. "Bond Graph 
Modeling of Marine Seismic Cables”. In ICBGM'2005

- International Conference on Bond Graph Modeling and Simulations.

[12] Rokseth, B., Skjong, S., and Pedersen, E., 2017. "Modeling of Generic Offshore Vessel in Crane Operations With Focus on Strong Rigid Body Connections". IEEE Journal of Oceanic Engineering, 42(4), oct, pp. 846868.

[13] Skjong, S., and Pedersen, E., 2014. "Modeling hydraulic winch system". In 2014 International Conference on Bond Graph Modeling and Simulation (ICBGM 2014), Vol. 46, pp. 181-187.

[14] Baumgarte, J., 1972. "Stabilization of constraints and integrals of motion in dynamical systems". Computer Methods in Applied Mechanics and Engineering, 1(1), pp. 1-16.

[15] Ostermeyer, G.-P., 1991. "On Baumgarte Stabilization for Differential Algebraic Equations”. In Real-Time Integration Methods for Mechanical System Simulation, E. J. Haug and R. C. Deyo, eds., Springer Berlin Heidelberg, pp. 193-207.

[16] Johansen, V., 2007. Modelling of flexible slender systems for real-time simulation and control applications.

[17] Johansen, V., Ersdal, S., Sørensen, A. J., and Leira, B., 2006. "Modelling of inextensible cable dynamics with experiments". International Journal of NonLinear Mechanics, 41(4), pp. 543 - 555.

[18] Skjong, S., and Pedersen, E., 2019. "On the numerical stability in dynamical distributed simulations". Mathematics and Computers in Simulation, 163, mar, pp. 183-203.

[19] Skjong, S., 2017. "Modeling and Simulation of Maritime Systems and Operations for Virtual Prototyping using Co-Simulations". Doctoral thesis, Norwegian
University of Science and Technology, Trondheim.

[20] Skjong, S., and Pedersen, E., 2017. "Co-Simulation of a Marine Offshore Vessel in DP-Operations including Hardware-In-the-Loop (HIL)". In Proceedings of the ASME 2017 36th International Conference on Ocean, Offshore, and Arctic Engineering OMAE 2017.

[21] Skjong, S., Kyllingstad, L. T., Reite, K.-j., Haugen, J., Ladstein, J., and Aarsæther, K. G., 2019. "Generic on-board decision support system framework for marine operations". In Proc. ASME 2019 38th Int. Conf. Ocean, Offshore and Arctic Eng.

[22] Sadjina, S., Skjong, S., Pobitzer, A., Kyllingstad, L. T., Fiskerstrand, R.-J., Torben, S., and Granholt, J. D. D. A., 2019. "Seismic RTDT-Real-time digital twin for boosting performance of seismic operations". In Proc. ASME 2019 38th Int. Conf. Ocean, Offshore and Arctic Eng.

[23] Fossen, T. I., 2011. Handbook of Marine Craft Hydrodynamics and Motion Control. John Wiley \& Sons, Ltd.

[24] Ersdal, S., 2004. "An Experimental Study of Hydrodynamic Forces on Cylinders and Cables in Near Axial Flow". Doctoral thesis; 2004:125, Norwegian Nniversity of Science and Technology, Trondheim.

[25] Blochwitz, T., Otter, M., Arnold, M., Bausch, C., Elmqvist, H., Junghanns, A., Mauss, J., Monteiro, M., Neidhold, T., Neumerkel, D., Olsson, H., Peetz, J.V., Wolf, S., and Clauss, C., 2011. "The Functional Mockup Interface for Tool independent Exchange of Simulation Models". In Proceedings of the 8th International Modelica Conference, Linköping University Electronic Press; Linköpings universitet, pp. 105-114.

[26] NTNU, and SINTEF Ocean, 2017. ViProMa www.viproma.no. 